\title{
Estructura urbana y policentrismo en el Área Metropolitana de Concepción ${ }^{*}$
}

\author{
CAROLINA ALEJANDRA ROJAS QUEZADA**, IVÁN MUÑIZ OLIVERA*** Y MI- \\ GUEL ÁNGEL GARCÍA-LÓPEZ**** \\ ** Departamento de Geografía, Universidad de Concepción. \\ *** Departamento de Economía Aplicada, Universidad Autónoma de Barcelona. \\ **** Departamento de Economía Aplicada, Universidad Autónoma de Barcelona.
}

\begin{abstract}
From the perspective of the functional model, the urban structure of the Metropolitan Area of Concepcion (Chile) is analyzed. The main objective is to identify and characterize the urban centers that comprise it as a result of the changes in the metropolitan population and its mobility, specifically with the use of employment indicators and density functions. The results approximate to an understanding of the hierarchical organization of the settlements and their centralities. This clarifies some of the patterns in the structure of the model, both monocentric and polycentric, which is a point of debate in discussions of metropolitan urban structures. The contributions of the research point to ways of describing and characterizing the behavior of the functional structure of the leading example of a metropolitan model in the south of the country.
\end{abstract}

KEYWORDS: urban structure, polycentricity, the Metropolitan Area of Concepción.

RESUMEN Desde la perspectiva del modelo funcional se analiza la estructura urbana del Área Metropolitana de Concepción (Chile). Principalmente se identifican y caracterizan los centros urbanos que la conforman en función la población y la movilidad metropolitana; específicamente se aplican indicadores de empleo y funciones de densidad. Los resultados permitieron una aproximación al entendimiento de la organización jerárquica de los asentamientos y las centralidades; aclarando algunas tendencias de la estructura del modelo, ya sea del tipo monocéntrico o policéntrico, tema en discusión si se refiere al análisis de las estructuras urbanas metropolitanas. Los aportes de la investigación apuntan a reflexionar sobre cómo se puede describir y caracterizar el comportamiento de la estructura funcional del modelo metropolitano por excelencia del sur del país.

PALABRAS CLAVE: estructura urbana, policentrismo, Área Metropolitana de Concepción.

\footnotetext{
Desarrollado en el marco de la Tesis Doctoral del autor principal, titulada "Análisis de la sostenibilidad de los modelos territoriales metropolitanos: el caso de Concepción (Chile). Aplicación de las tecnologías de información geográfica”. Financiado con la beca de postgrado CONICYT, Gobierno de Chile.

Recibido el 9 de septiembre de 2008, aprobado el 17 de marzo de 2009.

Correspondencia: Carolina Alejandra Rojas Quezada, Departamento de Geografía, Universidad de Concepción, Victoria s/n, Facultad de Arquitectura, Urbanismo y Geografía, Concepción, Chile. E-mail: crojasq@udec.cl. Iván Muñiz Olivera y Miguel Ángel García-López, Departamento de Economía Aplicada, Universidad Autónoma de Barcelona, Edificio B, Facultad de Ciencias Económicas, 08193 Belaterra, Barcelona, España. E-mail: ivan.muñiz@ uab.cat, miguelangel.garcia@uab.cat.
} 


\section{Las Áreas Metropolitanas: del monocentrismo al territorio en red}

Los cambios económicos, sociales y la urbanización de un territorio generalmente van estrechamente ligados al desarrollo industrial. En términos simples, estos avances traen como consecuencia un acelerado aumento de la población urbana, una extensión de su tamaño, más el surgimiento de nuevos fenómenos como la migración campo-ciudad y el desarrollo de sistemas de transporte cada vez más complejos. Estos factores han hecho que la ciudad se transforme en un espacio dinámico y en continuo crecimiento.

Al estudiar un Área Metropolitana lo habitual es analizar un conjunto de centros de diferente tamaño, funcionalmente integrados, cuestionándose el cómo se forma, cómo se articula y a qué debe su especial organización. La expansión urbana y la complementariedad de funciones entre los centros han generado un territorio metropolitano o agrupaciones de núcleos que pueden organizarse en distintos tipos de estructuras. Así se entendería que: un área metropolitana es un centro social, económico, político y administrativo, con núcleos urbanos estrechamente relacionados entre sí, conformando una unidad funcional. Entre estos obviamente existen flujos y especializaciones de bienes y servicios, que dan forma a una organización jerárquica de las ciudades, donde cada una tiene una función más desarrollada que la otra, generando un sistema interdependiente. Algunas funciones pueden conformar ciudades dormitorio, distritos industriales, comerciales y administrativos, pero todas están relacionadas en un conjunto integrado a través de los mercados de trabajo y vivienda.

$\mathrm{Al}$ intentar comprender la funcionalidad y las centralidades de un área metropolitana, es decir, su estructura parece imposible obviar el aporte de las clásicas teorías de la localización. En este sentido la explicación de las dinámicas de distribución de los uso de suelo comienza con Von Thünnen (1826), con su "Teoría de los Anillos Concéntricos", que representa la concentración de los distintos usos agrícolas alrededor de un centro de mercado. La "Teoría de la Localización Industrial” de Weber (1909) expresa la relación entre la localización de los recursos (materias primas) y el mercado mediante un triángulo, concluyendo que la instalación de una industria depende de la distancia a los recursos y al mercado. En los ańos veinte, Retcliffe (1925) analiza la centralidad en base a la influencia de las rentas en la distribución de los usos del suelo, y Burgues (1923), con su "Teoría Concéntrica", plantea la noción de que el desarrollo de la ciudad tiene lugar hacia fuera y a partir de su área central de servicios y comercio, formando una serie de coronas, anillos o áreas concéntricas. Más tarde, Hoyt (1939) propone la "Teoría de los Sectores" de la cual se deduce que el crecimiento de la ciudad comenzaba desde el centro, pero dependía de los ejes de transporte. Los avances conceptuales evolucionaron al desarrollo de una de las teorías más importantes del urbanismo moderno en relación a la organización urbana, la conocida "Teoría de los Lugares Centrales" (1933) creada por el geógrafo alemán Walter Christaller. Ésta básicamente consiste en que los asentamientos urbanos se sitúan en un espacio isotrópico a distancias regulares en torno a centros de servicios, por tanto el costo de un producto dependerá de factores como la distancia y el precio del transporte. En este marco, un lugar central es aquel núcleo de población que ofrece más bienes y servicios, siendo la cercanía a ellos por parte de los clientes el factor más importante que guía la distribución de los asentamientos urbanos (Wilson, 2000, pp. 118-151). Siendo un gran aporte el hecho de establecer la forma en que diversos factores se articulan en el territorio, dando lugar a una 
jerarquía urbana, fue una forma acertada de caracterizar las áreas funcionales por medio del establecimiento del grado de centralidad de los asentamientos que depende de los bienes y servicios que se oferten. Las tesis posteriores se enfocaron en seguir perfeccionando la explicación de la complejidad de la ciudad; la más visionaria fue la desarrollada por los geógrafos Harris y Ullman en 1945, la llamada “Teoría de Núcleos Múltiples”. Dichos autores plantearon que las ciudades tienen una estructura esencialmente celular, en la cual los diferentes tipos de utilización de suelo se han desarrollado alrededor de ciertos puntos de crecimiento o núcleos, situados en el interior del área urbana; expertos como Hall (2007) han seńalado que son los primeros en reconocer una estructura policéntrica en la ciudad.

En los últimos años, las aproximaciones teóricas enfocadas a entender la organización espacial metropolitana se basan en explicaciones sobre el comportamiento principalmente de variables económicas. La discusión actual se centra en conocer si las áreas metropolitanas en crecimiento, mantienen los clásicos modelos monocéntricos o tienden a modelos híbridos o absolutamente policéntricos. Es importante conocer si los modelos conceptuales reflejan realmente el comportamiento de las actuales áreas metropolitanas (Schwanen, Dieleman y Dijst, 2001).

En este sentido se ha mencionado que en las ciudades pequeñas el modelo monocéntrico puede constituir una buena aproximación (Redfearn, 2007), pero el modelo teórico de referencia actualmente ha pasado a ser el policéntrico, más aún cuando se ha asociado a la dispersión del crecimiento urbano. Antes se mencionó al modelo teórico de Harris y Ullman (1945) como el primero en sugerir que la ciudad podría convertirse en policéntrica. Hoy en día, evidentemente las grandes ciudades cada vez se hacen más policéntricas y los modelos clásicos antes presentados de hace 70 años, basados en la ciudad monocéntrica (excepto Harris y Ullman), han sido paulatinamente dejados de lado (Hall, 2007, p. 36).

La estructura clásica o monocentrismo simplemente se entiende desde la teoría de Von Thünnen, que básicamente explica la distribución de los usos del suelo alrededor de un centro principal. En cambio el policentrismo aplicado a un área metropolitana es un fenómeno nuevo, siendo aquel "proceso mediante el cual un área metropolitana se aleja paulatinamente de una estructura espacial caracterizada por la existencia de un solo centro de empleo, dirigiéndose hacia una nueva donde coexisten varios centros del mismo, originados por la descentralización del empleo o integración de centros pre-existentes" (Muñiz, Galindo y García López, 2005, p. 3). "Los subcentros de empleo desarrollan economías de escala y de aglomeración que desde un centro incrementan la proximidad al consumidor logrando dispersarse, cada centro significa una desconcentración del empleo, un punto para acceder al trabajo, al comercio y a la recreación" (Becerril-Padua, 2000, p. 5).

En esta discusión claramente se asume que las formas urbanas de las áreas metropolitanas están cambiando y que existe una tendencia a que muchas ciudades en expansión se alejen paulatinamente del monocentrismo, conformando estructuras policéntricas en las que coexisten diferentes subcentros. Si este fenómeno se suma a expresiones físicas del crecimiento urbano como la discontinuidad espacial y la baja densidad, entonces a menudo la policentralidad es etiquetada como dispersión, ya que no existe una precisa línea divisoria entre el policentrismo y la dispersión (García-López y Muñiz, 2007). Los cambios producidos por 
el crecimiento de la economía y la globalización se observan en ciertas ciudades y es interesante distinguir la expresión americana de la europea. Por ejemplo, la descentralización del empleo se refleja fuertemente en las ciudades norteamericanas, que en sí mismas se convierten en áreas metropolitanas, difusoras del modelo policéntrico, a menudo caracterizadas como dispersas. Por ejemplo, el área metropolitana de Los Ángeles en EE.UU., siempre citada como precursora de la ciudad policéntrica, debido al esparcimiento de los lugares de trabajo y servicios, separándose de los tradicionales centros y las viejas áreas metropolitanas de New York, Philadelphia, Chicago y Boston (William y Clark, 2000). En EE.UU., este fenómeno comienza cuando aparecen subcentros en la periferia, colonizando espacios vacíos u ocupados por viviendas con densidades bajas. Simplemente los lugares estratégicos de la periferia (nodos de transporte, lugares con ammenities ambientales, etc.) recogen algunas de las actividades que el CBD (Central Business District) expulsa (Muñiz, Galindo y García-López, 2005). Desde la visión europea se propone el desarrollo policéntrico como guía para conseguir un desarrollo territorial sostenible, más cohesionado económica y socialmente a gran escala, sugiriendo un sistema equilibrado y policéntrico entre ciudades y una nueva relación entre campo y ciudad (Comisión Europea, 1999).

Tanto en Europa como en Estados Unidos, el estudio de las regiones urbanas policéntricas no sólo ha derivado en la aparición de nuevos modelos teóricos, más complejos y refinados que el modelo monocéntrico. También ha aparecido un buen número de trabajos aplicados que básicamente han perseguido dos objetivos: en primer lugar, buscar algún método objetivo, simple y replicable que permitiese identificar los centros y/o subcentros; y en segundo lugar, contrastar algunas de las predicciones que se desprenden de los modelos teóricos de referencia. En el caso latinoamericano, aunque existe una rica tradición en el estudio de la composición, estructura y dinámica de las áreas metropolitanas, pocas veces se ha orientado la investigación utilizando el instrumental teórico y aplicado desarrollado en EE.UU. y Europa más actual. No se trata de aceptar acríticamente y más allá del contexto analizado un modelo teórico como hegemónico, sino de utilizarlo como trampolín para estudiar, con unos métodos cada vez más estandarizados, la estructura urbana de las metrópolis latinoamericanas.

El objetivo de la investigación que aquí se desarrolla es doble. Primero, identificar con algunos de los métodos utilizados en la literatura internacional consultada, los centros del Área Metropolitana de Concepción, en Chile. En segundo lugar, contrastar si el policentrismo detectado funciona tal como predice el modelo en cuanto al comportamiento de la densidad de población. ¿Alejarse del centro y de los subcentros implica una suave caída progresiva en los niveles de densidad?

\section{El Área Metropolitana de Concepción}

El Área Metropolitana de Concepción (en adelante AMC), localizada al centro sur de Chile $\left(36^{\circ} 35^{\prime}\right.$ y $37^{\circ} 00^{\prime}$ de latitud sur y los $72^{\circ} 45^{\prime}$ a $73^{\circ} 15^{\prime}$ de longitud oeste), es un territorio costero, cuya dinámica de crecimiento urbano ha despertado un especial interés científico plasmado en recientes publicaciones (Vásquez, Riveros y Romero, 2005; Rojas, Azócar, Vega, Kindler y Kabish, 2006; Pauchard, Aguayo, Peña y Urrutia, 2006; Pérez y Salinas, 2007). Su alcance funcional resulta bastante complejo: por un lado su más reciente delimitación procedente del 
Atlas Socio Ambiental, la define como un conglomerado urbano que se compone de 6 comunas (Concepción, Chiguayante, Hualpén, Penco, San Pedro y Talcahuano), representando un núcleo central conurbado y muy urbanizado (Rojas et al., 2006). En cambio para el Instituto Nacional de Estadísticas está compuesta por 9 comunas, las anteriormente mencionadas más Coronel, Lota y Tomé, así en su conjunto representan un núcleo urbano con más de 800 mil habitantes (Gobierno de Chile-Ministerio de Vivienda y Urbanismo MINVU, 2006); por último para el Plan Regulador Metropolitano de Concepción, es un territorio funcional y jerarquizado que se conforma por 11 comunas aparentemente muy relacionadas que concentran un volumen de población superior a los 500 mil habitantes (Gobierno de Chile - Ministerio de Vivienda y Urbanismo MINVU, 2003).

Por la diversidad de centros y su carácter más integrador se considera esta última delimitación, que incluye un borde costero de $60 \mathrm{~km}$., comprendidos entre el límite norte de la comuna de Tomé hasta el límite sur de la comuna de Lota, incluyendo en su totalidad a las comunas de: Concepción, Coronel, Chiguayante, Hualpén, Hualqui, Lota, Penco, San Pedro de la Paz, Santa Juana, Talcahuano y Tomé.

El territorio de análisis totaliza una superficie de 2.830,40 $\mathrm{km}^{2}$ representando el 7,63\% de la superficie regional. Según el Censo de Población y Vivienda (2002), cuenta con una población de 902.712 habitantes, equivalentes al 48,49\% de la población total, y el 57,31\% de la población urbana de la región del Bío-Bío.

La población actual reside principalmente en zonas urbanas (97\%) caracterizadas por ciudades intermedias emplazadas sobre las planicies costeras (terrazas fluviovolcánicas) a las riberas del río Bío-Bío, conviviendo con playas, dunas, acantilados rocosos, áreas de marismas, desembocaduras, humedales, bahías, penínsulas, islas, un golfo y la cordillera de la costa, con una densidad de 318,9 habitantes por $\mathrm{km}^{2}$ (INE, 2002). Los centros de Concepción y Talcahuano destacan como los principales articuladores urbanos de este espacio eminentemente industrial, como lo demuestra la distribución de la población económicamente activa (277.327 habitantes). Su población se dedica principalmente al comercio $(19,24 \%)$, industria manufacturera $(14,69 \%)$, actividades inmobiliarias y empresariales $(8,83 \%)$, construcción $(8,12 \%)$ y enseñanza o educación $(7,92 \%)$.

\section{Metodología}

Respondiendo al doble objetivo del trabajo, se aplicarán dos metodologías diferentes. La primera, una vez discutidas las alternativas disponibles, será utilizada para la identificación de los centros del sistema urbano. La segunda está orientada a contrastar el efecto de los subcentros (la distancia que los separa de cualquier localidad) sobre la densidad de población de dicha densidad. La identificación de subcentros no requiere de una extensa explicación, ya que a pesar de la multiplicidad de métodos surgidos recientemente, el estado del arte parece converger hacia la idea de que, preservando la máxima objetividad posible, el método idóneo dependerá de los datos disponibles, su escala espacial y el contexto en un sentido amplio de la realidad estudiada. 
El segundo objetivo del trabajo es contrastar el efecto de los subcentros sobre el comportamiento de la densidad de población. Según los modelos de la Nueva Economía urbana, CBD y subcentros se caracterizan ante todo por la concentración de puestos de trabajo. Dicha concentración en un número limitado de lugares, surge de la necesidad de estar en una ubicación estratégicamente escogida para captar el máximo mercado posible, o bien de acceder con un bajo coste a las economías de aglomeración que allí se originan. Una unidad residencial, cuanto más alejada esté de las principales concentraciones de empleo, mayor será la probabilidad de que tenga que afrontar unos importantes costes de desplazamiento. Para compensar dicho coste, tenderán a ofrecer (pujar) menos por esa vivienda, por lo que la renta del suelo ocupado será menor que en una localización cercana a los puestos de trabajo. El resultado final es que, al tener un menor precio, ese suelo se urbaniza con menor intensidad y con ello, la densidad de población será por tanto menor. Desde esta perspectiva el policentrismo no es sólo una cuestión de grumos de empleo, sino ante todo de su capacidad para orientar el comportamiento del precio del suelo, la intensidad de uso y la densidad, tanto de población como de empleo.

Una de las limitaciones más evidentes del enfoque del policentrismo basado en los modelos de la Nueva Economía Urbana, es que los subcentros se definen como concentraciones de empleo. Se supone que dicha concentración implica: el lugar donde la renta del suelo es anormalmente elevada, funciona como atractor neto de población de los alrededores, y tiende a concentrar funciones tanto más específicas cuanto mayor es su tamaño. Sin embargo, estas hipótesis pocas veces se contrastan. Tampoco incorporan la información referente al tipo de sociedad propio de un entorno denso, ni al tipo de urbanismo que lo acoge. Esta nota crítica no implica desde nuestra perspectiva, negar la utilidad y validez de estos modelos, aunque eso sí, aceptando y siendo conscientes en todo momento de sus limitaciones, que no son pocas.

Desde el punto de vista metodológico, en los últimos años el análisis de la estructura urbana policéntrica en áreas metropolitanas principalmente se ha estudiado en relación a los cambios en la dinámica de la población (Champion, 2001), la estructura de empleos (Redfearn, 2007), la dispersión del empleo (García-López y Muñiz, 2007; Muñiz, García-López y Galindo, 2008), la distribución de los tipos de hogares (De Bartolomé y Ross, 2007) y la movilidad (Schwanen et al., 2001; Gutiérrez y García-Palomares, 2005).

En el estudio se consideran los métodos procedentes de la nueva economía urbana que sugieren analizar el efecto que ejercen sobre la localización y la densidad de empleos (García-López y Muñiz, 2007). Así, en el caso de las estructuras monocéntricas, la densidad tiende a decrecer suavemente desde el centro hacia la periferia. Por el contrario, en las policéntricas, se producen picos de densidad y renta en espacios periféricos a partir de los cuales la densidad y la renta cae, dando como resultado una función de densidad y renta dentada (William y Clark, 2000).

El método aquí propuesto en primer lugar identifica y caracteriza los lugares de empleo en centros principales y subcentros mediante un análisis de la distribución de la población ocupada y una jerarquización de flujos de movilidad laboral. Posteriormente se aplican funciones de densidad que explican el efecto que ejercen sobre la distribución de la población, que permiten 
contrastar la hipótesis del monocentrismo o disminución de la densidad de centro a periferia (García-López y Muñiz, 2007; Muñiz et al., 2008).

\section{Identificación y caracterización de los centros urbanos}

Debido a la trascendental aportación de la teoría de Christaller, una de las primeras formas de caracterizar las áreas funcionales ha sido por medio de la identificación de los lugares centrales en los asentamientos, los cuales dependen de los bienes y servicios que oferten. En la práctica, éstos se han podido determinar mediante la medición de la concentración de establecimientos, servicios centrales y la atracción de viajes. Por ejemplo, en el Atlas Ambiental de Buenos Aires (2007), dicha caracterización se lleva a cabo mediante el estudio de la presencia bancaria, considerada como una actividad altamente asociada al comercio y la prestación de servicios, asumiendo que el espacio urbano es el producto de las actividades humanas de un proceso evolutivo de diferenciación y articulación funcional. Otra aproximación ha sido determinar la zona de influencia o radio de influencia de un asentamiento mayor, para el cual se han probado métodos como las encuestas de movilidad origen-destino, el comportamiento de los intercambios de información y los modelos gravitatorios.

$\mathrm{Al}$ intentar contrastar la hipótesis de monocentrismo metropolitano y no intraurbano en el área metropolitana de Concepción, se identifican los lugares concentradores del empleo con datos censales y de movilidad. Considerando que en gran parte los estudios situados en grandes áreas metropolitanas se desarrollan con métodos de identificación basados en umbrales, por ejemplo cantidad de puestos de trabajo localizados y densidades (Giuliano y Small, 1991; Giuliano y Small, 1999; Craig y Ng, 2001; De Bartolomé y Ross, 2007; Horner, 2007 y Redfearn, 2007). Concepción como un área de tamaño medio con solo once territorios y con centros cívicos importantes para el modelo de crecimiento urbano, se considera poco apropiado aplicar una metodología de umbrales por tanto se reconoce a los centros cívicos (CBD) como los centros de empleo o principales atractores de la población ocupada. En su caracterización se utilizan estadísticas censales del año 2002 e índices de densidad, autocontención y especialización económica (Ver anexos).

Con los datos disponibles, la aproximación a los puestos de trabajo se realiza consultando la información censal de situación laboral de la semana previa y comuna donde trabaja. La primera variable permite calcular a los ocupados (empleados), o la población en las situaciones de: trabajando por ingreso, sin trabajar pero tienen empleo y trabajando para un familiar sin pago en dinero. Por tanto con los datos de comuna donde trabaja se obtiene un número aproximado de puestos de trabajo localizados, ya que se calculan sólo los ocupados residentes que trabajan en la comuna. Como fuerza laboral, se considera a las personas capacitadas para trabajar, es decir, los ocupados más los desocupados. Para la población que no trabaja en su centro se desconoce hacia dónde se dirigen, ya que el censo no especifica el lugar, por tanto se establece una correlación con los datos de movilidad laboral de la encuesta origen-destino del año 1999 (SECTRA, 1999). Se modela el comportamiento de los viajes laborales, obteniendo los tres más importantes. Con los resultados de los índices anteriores de empleo y el ranking de flujos, se clasifican los centros cívicos en: principales y subcentros. A partir de la clasificación de los centros se propone una jerarquización en cuatro tipos: primer orden o lugares centrales, 
segundo orden o municipios integrados, tercer orden o comunas dormitorio y cuarto orden, correspondiente a los centros rurales.

\section{Modelo monocéntrico o policéntrico}

La organización e influencias que ejercen los centros principales y los subcentros se analiza mediante funciones teóricas de densidad, éstas permiten identificar bajo qué modelo se estructura el territorio. Las mencionadas funciones se basan en la medición de las distancias o medida de accesibilidad espacial desde cada área censal hacia el centro principal (CBD) y los subcentros (SUB). Si la densidad viene afectada significativamente y de forma negativa por la distancia a dichas concentraciones de empleo, el coeficiente estimado llamado gradiente de densidad será negativo y significativo (García-López, 2008). Estas funciones además han demostrado resultados excelentes en modelos metropolitanos como en el Área Metropolitana de Barcelona (García-López y Muñiz, 2007; Muñiz et al., 2008).

En este caso se utilizan dos ecuaciones lineales en función del comportamiento de la densidad según se aleja de los centros principales y los subcentros. La función que explica una estructura monocéntrica considera como variable dependiente al logaritmo neperiano de la densidad (lineal) y como variable independiente a la distancia al centro principal (CBD). Por tanto se cumple siempre y cuando el gradiente disminuya a medida que se aleja del centro principal y el resultado sea estadísticamente significativo.

Ec. (1)

$\ln ($ Den $)=c+\gamma_{C B D} d i s t_{C B D}$

En cambio, la función que explica una estructura policéntrica considera como variables independientes a la distancia al centro principal (CBD) y a la menor distancia de las calculadas hacia los subcentros (SUB). Si el modelo resulta significativo, se valida la hipótesis de policentrismo, explicando que los subcentros son tan importantes como el centro.

Ec. (2)

$$
\ln (\text { Den })=c+\gamma_{C B D} d i s t_{C B D}+\gamma_{S U B} d i s t_{S U B}
$$

C es la densidad teórica central, $\gamma$ CBD es el gradiente de densidad del CBD y $\gamma \mathrm{SUB}$ el gradiente de densidad del subcentro. Con su aplicación se intenta explicar si el territorio se estructura bajo un modelo monocéntrico o policéntrico. Desde los aspectos más técnicos las ecuaciones se calculan utilizando herramientas de econometría y de sistemas de información geográfica (SIG), estos últimos han sido de gran aporte debido a que permiten calcular las distancias entre los centros y subcentros. Actualmente además resultan apropiados en estudios de localización de puestos de empleos (Redfearn, 2007) y de viajes laborales derivados de la localización de los puestos de trabajo (Horner, 2007). Como sugiere Redfearn (2007), las técnicas SIG se consideran un aporte para el desafío de desarrollar métodos que permitan entender la complejidad del cambio y la evolución de un modelo monocéntrico a modelos policéntricos. 


\section{Resultados}

\section{Distribución y organización de los asentamientos}

El AMC es un área de reciente formación que desde mediados del siglo pasado comienza a constituir una aglomeración urbana central, producto de un crecimiento de población influenciado por la concentración de actividades industriales. Los principales centros poblados son Concepción, Talcahuano y Coronel, ninguno de ellos supera los 300.000 habitantes; los dos primeros, por tradición histórica, son los grandes articuladores del territorio.

La distribución de los centros es aleatoria $(\mathrm{Rn}=1,21)^{1}$, el radio es de unos $45 \mathrm{~km}$. incluyendo a la comuna de Santa Juana. La primera corona metropolitana se muestra más bien concentrada y conurbada, orbitando alrededor de Concepción y Talcahuano los municipios de Hualpén, Chiguayante, San Pedro y Penco; la segunda corona la representan las ciudades desarrolladas a lo largo del borde costero, con áreas urbanas más pequeñas y con tradición industrial, portuaria; mereciendo especial atención el subsistema Coronel-Lota, municipios históricamente vinculados por sus actividades económicas ligadas a la minería. Por último se ubican los municipios de extensas superficies rurales, cuya integración a los centros principales es muy dependiente de las infraestructuras de transporte (Figura 1).

\footnotetext{
1 Medida de distribución espacial de los asentamientos $\mathrm{R} n=2 * \frac{d \sqrt{N}}{S}$, dónde d es la distancia, $\mathrm{N}$ el número de asentamientos y $S$ la superficie total. Rn oscila entre 0 y 2,15, siendo 0 una distribución concentrada y 2,15 uniforme.
} 
Figura 1

SISTEMA DE ASENTAMIENTOS Y CORONAS METROPOLITANAS

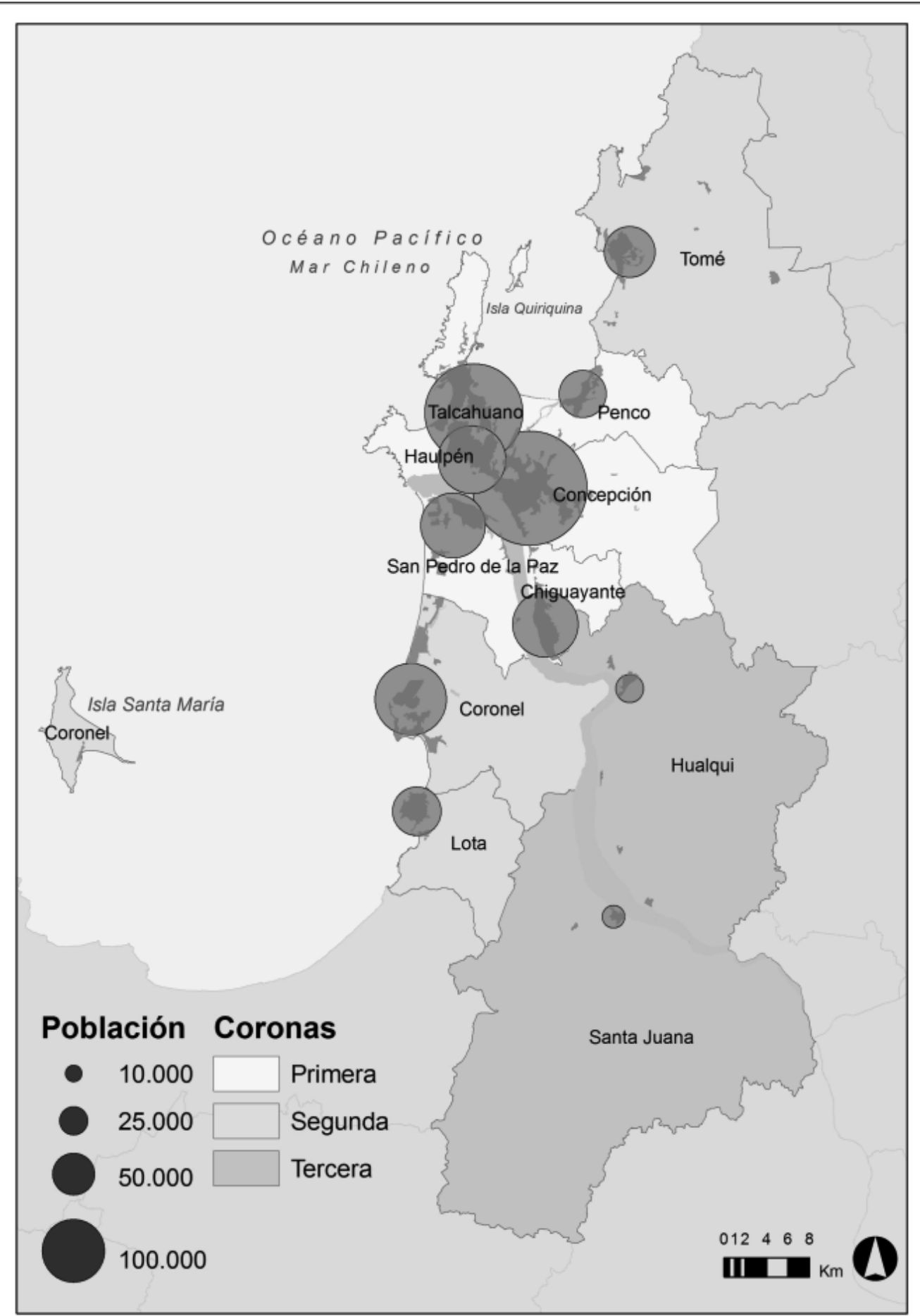

Fuente: Elaborado con datos del Censo de Población y Vivienda, 2002 
En general, los centros y subcentros poseen densidades de población ocupada muy bajas. Talcahuano es la comuna con mayor densidad, específicamente 3,2 ocupados por há (incluido Hualpén), en cambio las más bajas se localizan en las ciudades más pequeñas y rurales como Hualqui y Santa Juana, dadas las extensas superficies y la poca población. Los niveles de autocontención más bajos se encuentran en Chiguayante y San Pedro de la Paz, lo que hace suponer un modelo con mayor cantidad de población que se desplaza a otras comunas para trabajar, mientras que los niveles más altos están en Concepción, Santa Juana y Tomé (Cuadro 1).

Cuadro 1

EVOLUCIÓN DE LA POBLACIÓN EN EL CONJUNTO DE LA AGLOMERACIÓN

\begin{tabular}{|c|c|c|c|c|c|c|c|c|}
\hline \multirow[b]{2}{*}{ AMC } & \multirow{2}{*}{$\begin{array}{l}\text { Fuerza } \\
\text { Laboral }\end{array}$} & \multirow{2}{*}{$\begin{array}{c}\text { Población } \\
\text { Ocupada } \\
\text { Total }\end{array}$} & \multicolumn{3}{|c|}{ Ocupados } & \multirow[b]{2}{*}{ Otros } & \multirow[b]{2}{*}{ Densidad } & \multirow{2}{*}{$\begin{array}{c}\text { Auto- } \\
\text { contención }\end{array}$} \\
\hline & & & Total & $\begin{array}{c}\text { en la } \\
\text { comuna }\end{array}$ & $\begin{array}{r}\text { en otra } \\
\text { comuna }\end{array}$ & & & \\
\hline Chiguayante & 30818 & 26373 & 24492 & 7525 & 16967 & 1881 & 1 & 0,28 \\
\hline Concepción & 83816 & 72747 & 67857 & 52191 & 15666 & 4890 & 2,38 & 0,71 \\
\hline Coronel & 31538 & 26457 & 24368 & 16137 & 8231 & 2089 & 0,58 & 0,6 \\
\hline Hualqui & 6011 & 5004 & 4535 & 2010 & 2525 & 469 & 0,03 & 0,4 \\
\hline Lota & 15523 & 12669 & 11432 & 6834 & 4598 & 1237 & 0,59 & 0,53 \\
\hline Penco & 16499 & 13897 & 13196 & 5682 & 7514 & 701 & 0,52 & 0,4 \\
\hline San Pedro & 30741 & 26453 & 25197 & 8570 & 16627 & 1256 & 0,76 & 0,32 \\
\hline Santa Juana & 3540 & 3014 & 3014 & 2109 & 905 & 0 & 0,02 & 0,69 \\
\hline Talcahuano & 89504 & 76119 & 70543 & 47662 & 22881 & 0 & 3,26 & 0,62 \\
\hline Tomé & 17922 & 14636 & 13369 & 10349 & 3020 & 1267 & 0,2 & 0,7 \\
\hline
\end{tabular}

Fuente: Elaborado con datos del Censo de Población y Vivienda, 2002 Nota: Los resultados del indicador de ocupación se muestran en la Figura $\mathrm{N}^{\circ} 4$

La distribución del empleo relacionado a la población ocupada se concentra principalmente en la primera corona, dominada por los núcleos de Concepción y Talcahuano; éstos están rodeados por municipios con más empleados fuera de sus territorios (Figura 2). 
Figura 2

\section{DISTRIBUCIÓN POBLACIÓN OCUPADA RESIDENTE}

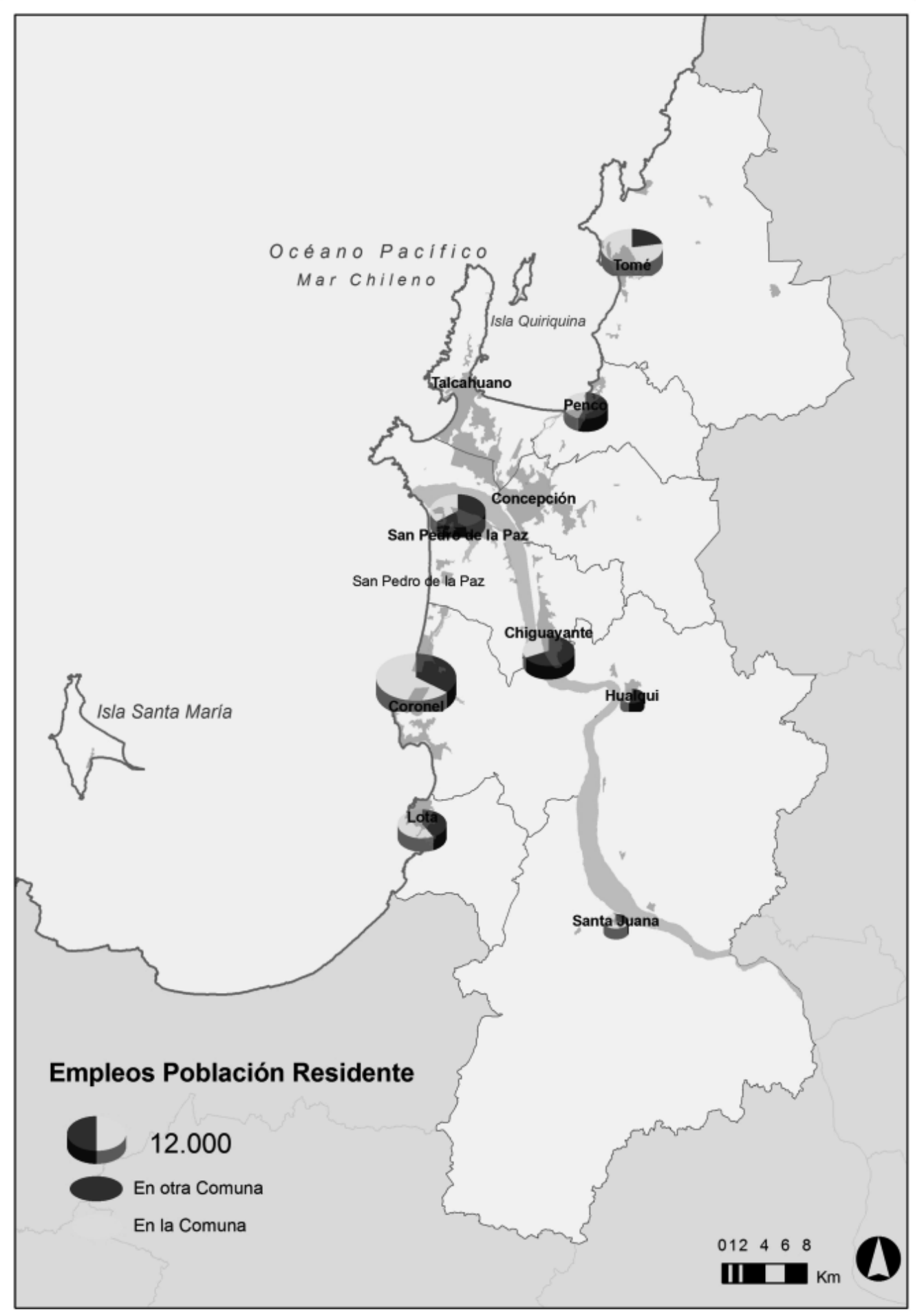

Fuente: Elaborado con datos del Censo de Población y Vivienda, 2002 
En la ocupación de la población se manifiestan la existencia de ciertas concentraciones que merecen atención: los municipios tradicionales costeros, con la excepción de Lota, mantienen una especialización industrial y actividades del secundario (industria manufacturera); los municipios nuevos, independizados de la administración de Concepción y Talcahuano, se caracterizan más por cumplir funciones ligadas a la prestación de servicios personalizados y profesionales; y los municipios rurales, a las ramas propias del sector primario. Las comunas de Talcahuano, Coronel y Tomé son las que tienen una distribución más equilibrada de su población ocupada, siendo a su vez los municipios con mayor localización industrial (Figura 3).

Figura 3

ESPECIALIZACIÓN FUNCIONAL

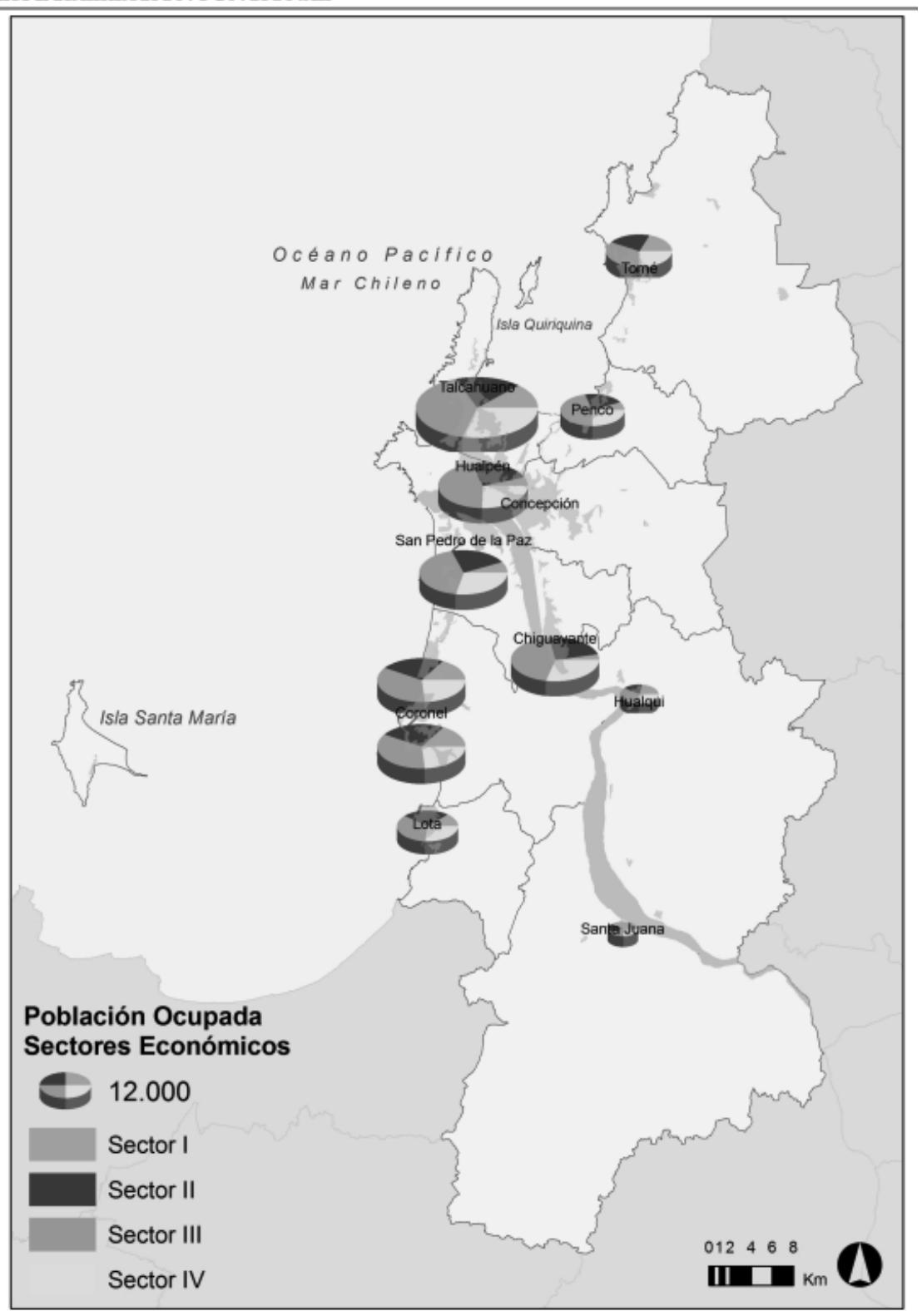

Fuente: Elaborado con datos del Censo de Población y Vivienda, 2002 Nota: Los gríficos muestran la distribución de la ocupación por sectores económicos 


\section{Jerarquias metropolitanas}

Los resultados expuestos hasta ahora sólo reflejan algunas características de los centros y subcentros, desconociendo cuáles de ellos ejercen como lugares centrales o ejercen un grado de atracción mayor. Por ello, se incorpora la movilidad metropolitana (EOD), donde el volumen de viajes permite analizar ciertos patrones: la conectividad se produce entre todos los centros, siendo Hualqui el municipio con menor participación e integración en la red metropolitana. La mayor proporción de viajes se dirige hacia la capital Concepción y proceden desde todos los centros. Dado que los índices planteados se refieren a la población ocupada se modelan solo los desplazamientos por propósito laboral y el número de empleados fuera de su comuna de procedencia, en caso de una relación estadística positiva ( 0,682 coeficiente de Pearson), se asume que los centros que absorben la mayor cantidad de flujos laborales tienen una jerarquía mayor, por tanto son más centrales que el resto (Figura 4). 


\section{FLUJOS PROPÓSITO LABORAL}

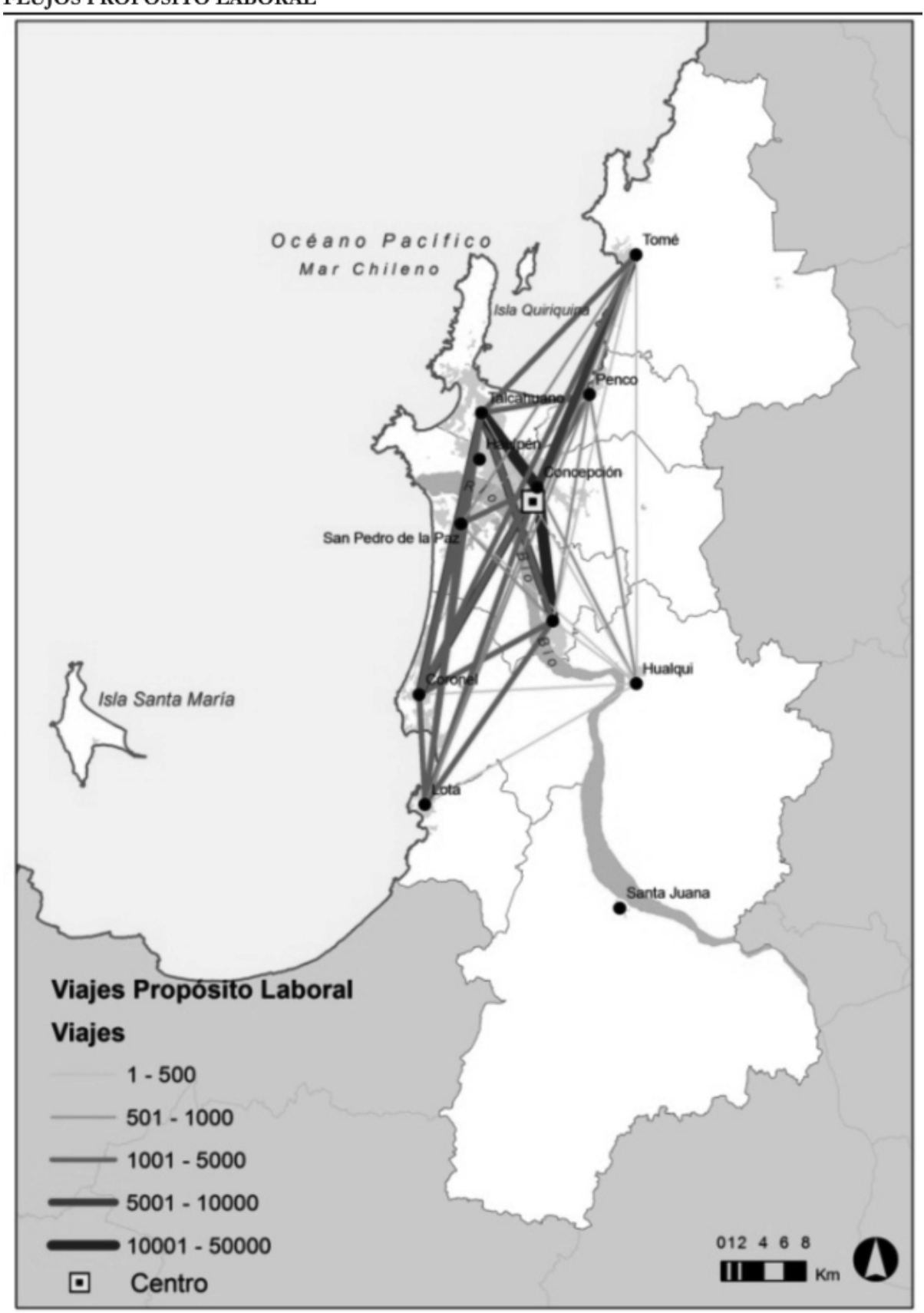

Fuente: Elaborado con datos de la EOD, 1999

El escenario indica que, sin duda, los principales generadores y atractores de flujos laborales son Concepción y Talcahuano, ciudades funcionalmente complementarias. Se destacan los casos de San Pedro y Chiguayante, o municipios creados en las últimas décadas. Éstos probablemente deberían ser ejemplos de descentralización del empleo, sin embargo son los que en mayor porcentaje sus empleados residentes trabajan fuera y tienen como destinos principales las ciudades de Concepción y Talcahuano. Hualqui es el asentamiento menos integrado, sus 
flujos se orientan hacia Concepción, Chiguayante y Talcahuano y su atracción en el sistema metropolitano es mínima comparada con el resto, cumpliendo más labores de municipio rural. Al desconocer los datos de Santa Juana, se asume un comportamiento muy similar.

En el marco de los resultados en los análisis de empleo, funcionalidad y movilidad metropolitana, se ordena y clasifica la región funcional en 4 tipos de centros metropolitanos: los de primer, segundo, tercero y cuarto orden (Figura 5).

- Primer Orden o Lugares Centrales: asentamientos intermedios mayores, presentan las densidades y los niveles de autocontención más altos, con valores superiores a los 10.000 puestos de trabajo ocupados por residentes. Son asentamientos especializados que desde el siglo pasado concentraban la población y la urbanización debido a la industrialización, por tanto ya se consolidaban como centros de empleo que se mantienen hasta hoy. A este grupo pertenecen Concepción y Talcahuano.

- Segundo Orden o Municipios Integrados: asentamientos menos diversos económicamente y muy industriales y portuarios, con bajas densidades y que en el pasado tuvieron mucho más dinamismo y especialización económica que en la actualidad. A este grupo pertenecen Lota y Coronel, relacionados con la industria del carbón, y Penco y Tomé, con la loza y los textiles.

- Tercer Orden o Municipios Dormitorios: asentamientos de origen administrativo muy reciente, cuyos procesos de crecimiento están muy vinculados a los municipios centrales. Son los grandes atractores del uso residencial con densidades de empleo más bajas que los de primer orden pero más altas que los de segundo. Localizados muy próximos a Concepción, poseen menor mezcla entre población y empleo y baja autocontención, traducida en desplazamientos de su población principalmente hacia Talcahuano y Concepción. Sus niveles de especialización son en el sector terciario y en otras actividades. A este grupo pertenecen Chiguayante y San Pedro de la Paz.

- Cuarto Orden o Centros Rurales: asentamientos rurales de menor envergadura, menos integrados en el funcionamiento metropolitano, con bajísimas densidades de empleo. Se ocupan principalmente en la agricultura. Su nivel de autocontención es medianamente alto. A este grupo pertenecen Santa Juana y Hualqui. 


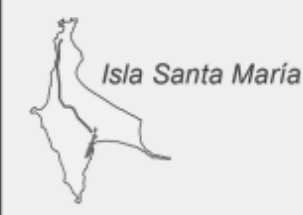

\section{Jerarquías Centros}

- Primer Orden

- Segundo Orden

- Tercer Orden

- Rurales Integrados

Vialidad Metropolitana

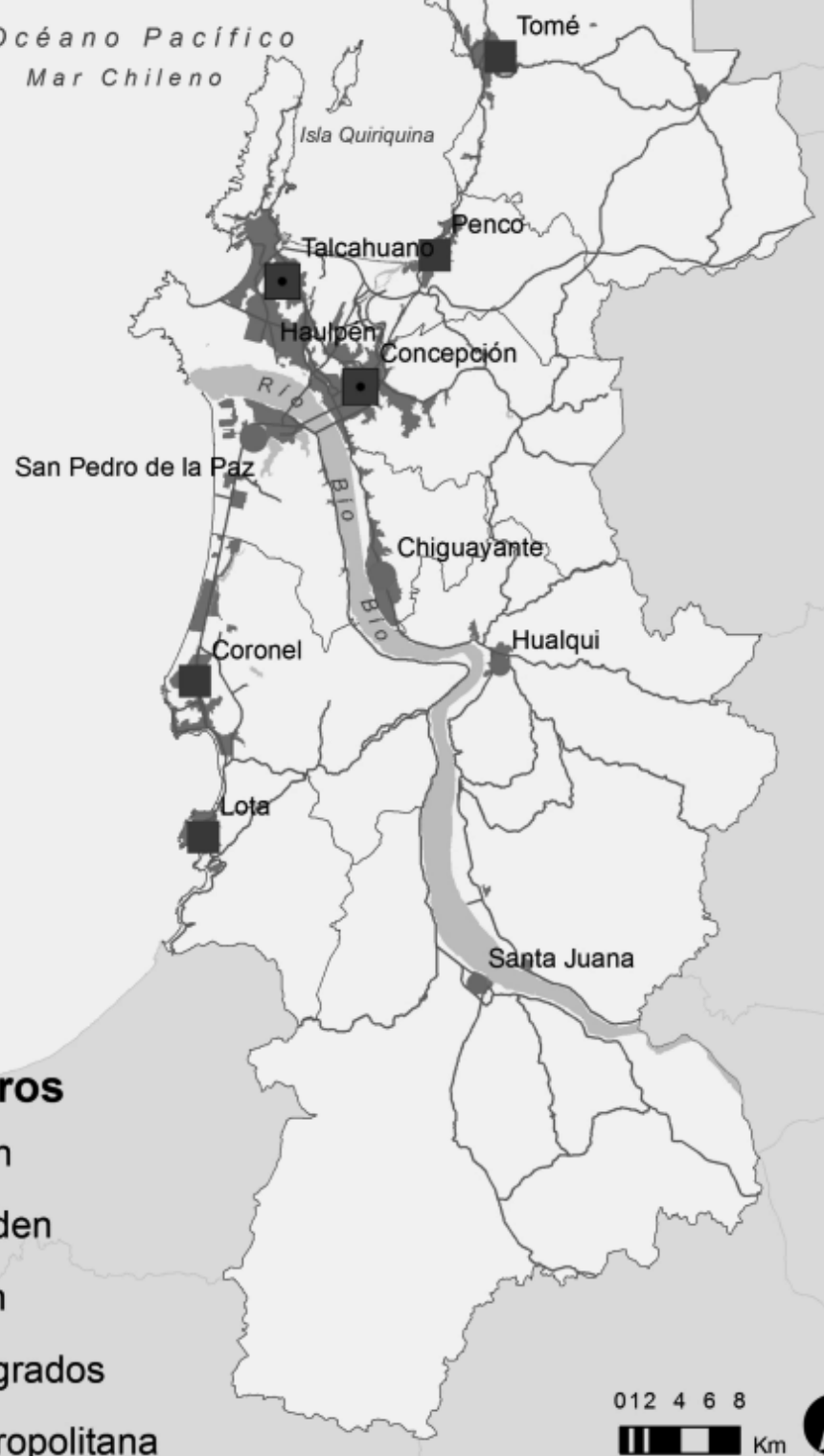

II $\mathrm{Km}$ 


\section{El modelo de desarrollo funcional de los centros ¿monocéntrico o policéntrico?}

De acuerdo a la jerarquía metropolitana, ha quedado demostrado que en el AMC la centralidad la ejercen las comunas de Concepción y Talcahuano (CBD); son los grandes atractores, polos de empleo y concentradores de las actividades económicas. Sus áreas de influencia cubren toda el área metropolitana y más bien en esta primera aproximación responden a un modelo bicéntrico o bicéfalo $(\mathrm{IP}=38)^{2}$, pero al tratarse de dos o más centros dominantes, se puede interpretar una tendencia hacia un modelo policéntrico. En los trabajos aplicados a la realidad chilena, el policentrismo en general se ha entendido como sinónimo de dispersión. Por ejemplo, la capital Santiago ha sido catalogada como una región suburbanizada, extremadamente segregada, fragmentada, policéntrica, con límites imprecisos, cuya dinámica expansiva es incorporar terrenos urbanos aledaños y rurales, configurando un extenso periurbano (De Mattos, 1999). Para Becerril-Padua (2000) el crecimiento urbano de Santiago dio lugar a formas policéntricas, las cuales se ven favorecidas por las normas del Plan Regulador Metropolitano que define subcentros en el interior del Área Metropolitana. Se observan fenómenos asociados a la ciudad dispersa, relación muy ligada a una visión americana del crecimiento urbano, que para la realidad europea pueden ser incluso hasta contrapuesta (García-López y Muñiz, 2007).

Como se ha descrito en el apartado sobre la metodología, la policentralidad puede describirse con el comportamiento de variables como: los flujos laborales, los precios de suelo y la densidad de población. En este trabajo se contrasta la hipótesis de policentrismo examinando el valor y significatividad del gradiente de densidad de la distancia al subcentro en una función de densidad de población, así como en la capacidad explicativa global del modelo $\left(\mathrm{R}^{2}\right)$ en comparación con una función monocéntrica. Si al incorporar como regresor la distancia a los subcentros su gradiente presenta el signo esperado, es significativo y aumenta la capacidad explicativa del modelo, la hipótesis de policentrismo se impone frente a la de monocentrismo (García-López y Muñiz, 2007).

La primera función o monocéntrica considera sólo los lugares centrales (Concepción y Talcahuano) y la segunda o policéntrica a éstos más la distancia más próxima a los subcentros o centros de segundo, tercer y cuarto orden.

Los resultados de las funciones de densidad se observan en el Cuadro 2. Para su mejor comprensión, se detalla que entre paréntesis se incorpora el valor de la $t$ de student.

2 Índice de Primacía $I P=\frac{P 1}{\sum_{i=1}^{4} P} * 100$ 


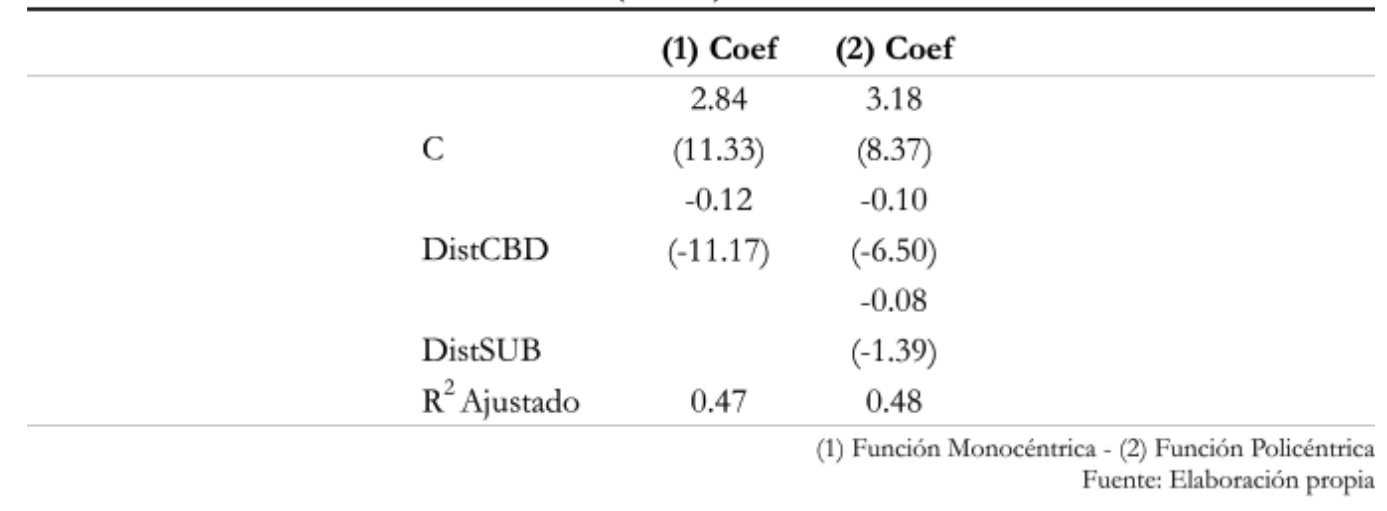

Los resultados permiten inferir que el AMC es un modelo cuya articulación del territorio sigue en función de los centros principales (primer orden). Aunque con el signo esperado, el gradiente del subcentros no supera un nivel de significatividad del $99 \%(\mathrm{t}=2)$ y la capacidad explicativa del modelo aumenta sólo muy ligeramente. Estos resultados parecen desmentir la vigencia del modelo policéntrico. Sin embargo, es posible que esto se deba a una mala especificación espacial del modelo. Uno de los problemas es que los dos centros principales están muy próximos, muy cercanos. Además, se supone que los subcentros ejercen un efecto espacial sobre los niveles de densidad de la misma dimensión que el CBD. Para corregir estos problemas, McDonald y Prather (1994) y García-López y Muñiz (2007) proponen un modelo diferente, donde, en lugar de utilizar la distancia directa al subcentro, se incluye la invertida. Esto permite acotar el área de influencia del subcentro y reducir los niveles de multicolinealidad.

Ec. (3)

$\ln ($ Den $)=c+\gamma_{C B D} \operatorname{dist}_{C B D}+\gamma_{S U B}\left(\frac{1}{\text { dist }_{S U B}}\right)$

Además, en la nueva ecuación, y para compensar la influencia de los centros principales, se utiliza la menor distancia a cualquiera de los dos centros (Concepción - Talcahuano).

Los resultados obtenidos de esta nueva especificación aparecen en el cuadro 3. Tal como puede apreciarse, la capacidad explicativa del modelo ha aumentado de forma significativa, y lo que es más importante el gradiente de densidad de los subcentros presenta el signo esperado (positivo al ser estimado en su forma inversa) y es estadísticamente significativo. 


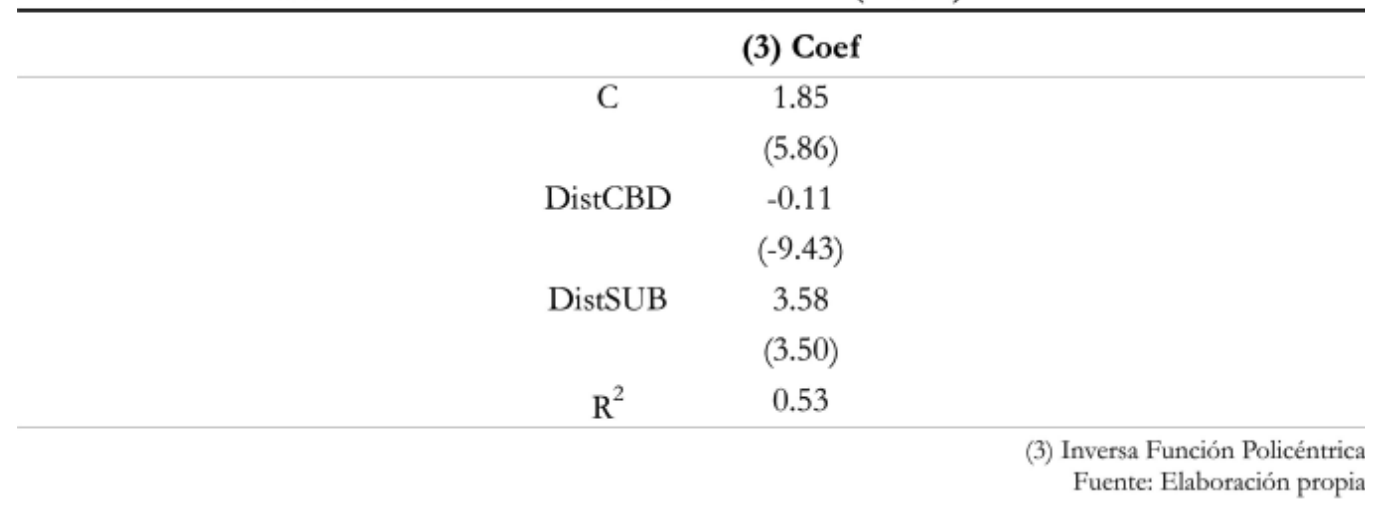

Esta estructura refleja un efecto más localizado sobre la densidad, es decir, muy condicionado al espacio próximo del subcentro o a su área de influencia; fuera de ese radio la articulación se sigue relacionando con la potencialidad de los centros principales, es decir, éstos no pierden relevancia como articuladores del territorio. Se definiría como un policentrismo espacialmente restringido cuyo ámbito de acción de los subcentros es menor. Por ejemplo, ciudades descentralizadas administrativamente, como San Pedro y Chiguayante, son atractivas para el uso residencial, pero a su vez muy dependientes de los lugares centrales, especialmente en puestos de trabajo y servicios.

En la escala de análisis, el policentrismo existe siempre y cuando en un sistema urbano de varias ciudades de distintos niveles existen dos o más ciudades que se complementan en diferentes funciones. Inferimos que históricamente el sistema analizado nunca ha funcionado como un modelo monocéntrico propiamente tal, aunque se reconoce que la influencia de Concepción tiende a mantenerse, debido a la importante concentración de servicios y equipamientos metropolitanos. Ambas comunas están absolutamente integradas y se complementan funcionalmente, aportando cada una sus especialidades. Por ejemplo, Talcahuano-Hualpén concentran la localización industrial, factor tan característico de un área metropolitana.

Considerando la idea del policentrismo como la alternativa a la dispersión, se define que en el AMC no existe un modelo policéntrico consolidado. Si existiese, habría un mayor equilibrio en el tamaño, población y en la especialización funcional del resto de los asentamientos.

\section{Discusión y conclusiones}

El Área metropolitana de Concepción ha sido descrita como policéntrica y multifuncional (Vásquez et al., 2005). Sin embargo, en este trabajo mediante una metodología estadísticamente validada, se define como una estructura funcionalmente bicéntrica, pero espacialmente como una estructura policéntrica restringida, debido a la acotada influencia de los subcentros en relación a los dos centros principales (Concepción y Talcahuano). 
La investigación profundiza en la estructura policéntrica desde la visión metropolitana, considerando los centros principales de cada comuna y no desde la escala intraurbana. En este sentido se considera que para futuras investigaciones será interesante incorporar como un centro independiente el eje de unión entre las comunas principales, el cual está adquiriendo un dinamismo considerable en cuanto a equipamiento, comercio, oferta de empleos y servicios.

Desde esta visión metropolitana, al considerar la estructura urbana policéntrica desde la teoría europea, es decir la policentralidad como una alternativa a la dispersión y la apuesta a un desarrollo más equilibrado y sostenible (Comisión Europea, 1999; Hall, 2007). El AMC no se entendería como una estructura consolidada y equilibrada, ya que evidentemente las mayores oportunidades se siguen concentrando dentro de sus dos centros principales (Concepción y Talcahuano). Según la visión de la teoría americana, la aparente dispersión de la mancha de aceite central, en general más típico del modelo monocéntrico, ha evolucionado de forma dispersa alrededor de Concepción; esto forzaría una asociación hacia una estructura más policéntrica. En cualquier caso, a menudo los términos de policentralidad, fragmentación y dispersión son tratados como sinónimos. Según los resultados obtenidos, estos conceptos están muy relacionados, pero en este caso no se incluye a la policentralidad como manifestación o consecuencia de la dispersión del crecimiento urbano.

Si el AMC apuesta al modelo policéntrico como la estructura más adecuada para conseguir un desarrollo territorial más equilibrado, ésta al menos debería apuntar a consolidar su sistema de transporte público, favoreciendo la caída de los costos de desplazamiento de la residencia al trabajo, consolidando como una alternativa real y eficiente que permita integrar estos nuevos suburbios de crecimiento. Esta estructura favorecería la complementariedad funcional, potenciando la distribución más equilibrada de las funciones metropolitanas, estimulando las redes urbanas y las sinergias entre las ciudades del Área Metropolitana.

Se considera que la investigación ha permitido evaluar el modelo territorial desde la mirada de la estructura funcional. Se ha hecho además con métodos que sirven para contrastar los resultados con otras áreas metropolitanas del país o del mundo. A pesar de ser diseñados para una zona en concreto, son perfectamente aplicables en otros territorios. Con respecto al caso particular estudiado, la investigación reconoce al territorio como un área metropolitana compleja, de reciente formación, cuyo crecimiento está muy condicionado por factores ambientales como el borde costero, la hidrografía y los cordones montañosos, y socioeconómicos como el aumento de la población. Por supuesto, es un territorio en profunda reestructuración, hecho que claramente se demuestra con la división en nuevas administraciones comunales.

A modo de conclusión final se especifica que es un modelo que:

- Posee una conformación territorial que se organiza en un sistema bicéntrico, específicamente un modelo policéntrico espacialmente restringido, en el cual los dos centros principales (Concepción y Talcahuano) ejercen como articuladores y dinamizadores del territorio, aunque se reconoce un impacto menor, espacialmente más localizado, de los subcentros o resto de las comunas integrantes. 
- Las fuerzas que están interactuando en la expresión de formas policéntricas, tienen que ver con el modelo centrífugo en el área central, es decir Concepción y Talcahuano crecieron de un modo que generó nuevas áreas residenciales cada vez más lejos de sus centros. Éstas comenzaron a crear sus propias dinámicas y centros de producción y servicios, por tanto, tienen que consolidarse como centros independientes (Penco, Chiguayante y San Pedro). Por otro lado, el modelo de incorporación se presenta en municipios de desarrollo histórico que antes fueron autosuficientes en empleo y algunos servicios, y hoy necesitan pertenecer a un núcleo mayor para atraer actividades no residenciales que permitan diversificar su funciones económicas (Coronel y Tomé).

- Se organiza como un sistema de centros de distinto tamaño, que en principio siguió el modelo de mancha de aceite propio de la ciudad monocéntrica, configurando una conurbación central. La población se distribuye en función de las distancias, dispersándose debido a la disminución de las densidades en periferias y distritos rurales de grandes extensiones territoriales.

- No se puede confundir esta tendencia de crecimiento disperso con la evolución hacia las formas policéntricas equilibradas, más aún cuando actualmente se está discutiendo si el desarrollo policéntrico es el que probablemente más se adecue al desarrollo de áreas metropolitanas compuestas por ciudades medias. En este sentido esta investigación ayuda a clarificar la diferencia entre un desarrollo policéntrico consolidado y las manifestaciones de un área conurbada de crecimiento periférico.

- Siguiendo la línea de investigación, en futuros trabajos será muy interesante aproximarse al estudio de la policentralidad incorporando como centro independiente el sector el Trébol y su entorno cercano. Éste como parte del eje divisorio entre las comunas principales que dada la instalación de centros comerciales, universidades y aeropuerto, está adquiriendo un fuerte dinamismo en cuanto a oferta de empleos, servicios y concentración de viajes. También la identificación de centros mediante la identificación de la oferta de bienes y servicios será un factor importantísimo para contrastar y comprobar estos resultados a una escala intraurbana.

Finalmente se considera que los principales aportes de la investigación tienen relación a la aplicación de metodologías que permiten analizar las estructuras funcionales de territorios de organización compleja, como las áreas metropolitanas de tamaño medio.

\section{Anexos}

Los índices aplicados para caracterizar los centros cívicos son:

- Densidad (PobOcup/ST), donde PobOcup corresponde a la población ocupada en la comuna y $S T$ a la superficie total de la comuna.

- Autocontención (PobOcup/PobOcupT), donde PobOcup corresponde a la población ocupada de la comuna y PobOcup T a la población ocupada total residente en la comuna. Apunta a la relación entre la población ocupada residente que trabaja en la misma comuna donde habita.

- Ocupación (PobOcupj/PobOcupT)*100, donde PobOcupj corresponde a la población ocupada total residente en la comuna. Apunta a la relación entre la población ocupada 
residente que trabaja en la misma comuna donde habita. Corresponde al porcentaje de población ocupada en cada uno de los sectores económicos $j$.

\section{Referencias Bibliográficas}

Agencia de Promoción Científica y Tecnológica de la Secretaria de Ciencia Tecnología e Innovación Productiva de la Nación (2007). Atlas ambiental de Buenos Aires. Recuperado el 10 octubre de 2007, de http://www.atlasdebuenosaires.gov.ar.

Becerril-Padua, M. (2000). Policentrismo en las ciudades latinoamericanas. El caso de Santiago de Chile. Theomai. Recuperado el 24 de julio 2007, de redalyc.uaemex.mx/redalyc/ pdf/124/12400108.pdf.

Comisión Europea (1999). Estrategia territorial europea. Hacia un desarrollo equilibrado y sostenible del territorio de la Unión Europea. Recuperado el 15 de junio 2008, de http:// ec.europa.eu/regional_policy/sources/docoffic/official/reports/pdf/sum_es.pdf.

Craig, S.G. \& Ng, P.T. (2001). Using quantile smoothing splines to identify employment subcenters in a multicentric urban area. Journal of Urban Economics, 49, 100-120.

Champion, A. (2001). A changing demographic regime and evolving polycentric urban regions: consequences for the size, composition and distribution of city populations. Urban Studies, 38, 4, 657-677.

De Bartolome, C. \& Ross, S.L. (2007). Community income distributions in a metropolitan area. Journal of Urban Economics, 61, 496-518.

De Mattos, C. (1999). Santiago de Chile, globalización y expansión metropolitana: lo que existía sigue existiendo. EURE, 25, 76, 29-56.

García-López, M-Á. \& Muñiz, I. (2007). ¿Policentrismo o dispersión? Una aproximación desde la nueva economía urbana. Investigaciones Regionales, 11, 25-43.

García-López, M-Á. (2008). Quince años de suburbanización en la Barcelona Metropolitana, ¿Se está dispersando la población? Investigaciones Económicas, 32, 1, 53-86.

Giuliano, G. \& Small, K. (1991). Subcenters in the Los Ángeles region. Regional Science and Urban Economics, 21, 163-182.

Giuliano, G. \& Small, K. (1999). The determinants of growth of employment subcenters. Journal of Transport Geography, 7, 189-201.

Gobierno de Chile - Secretaría Interministerial de Planificación de Transporte, SECTRA (1999). Encuesta de origen y destino de viajes del Gran Concepción. Recuperado el 10 de diciembre 2007, de http://sintia.sectra.cl/.

Gobierno de Chile - Ministerio de Vivienda y Urbanismo, MINVU (2003). Plan Regulador Metropolitano de Concepción. Memoria Explicativa.

Gobierno de Chile - Ministerio de Vivienda y Urbanismo, MINVU (2006). Diagnóstico Urbano 1990 - 2006. Recuperado el 28 de diciembre 2006, de http://www.observatoriourbano.cl/ docs/pdf/Diagn\%C3\%B3stico\%20Urbano\%20Completo\%20actualizaci\%C3\%B3n20\%20 mayo\%202007revisado\%20junio.pdf.

Gutiérrez, J. \& García-Palomares, J. (2005). Cambios en la movilidad en el área metropolitana de Madrid: el creciente uso del transporte privado. Anales de Geografía de la Universidad Complutense, 25, 331-351.

Hall, P. (Ed.) 2007. Congreso Regiones Capitales. Madrid: Comunidad de Madrid. 
Horner, M. (2007). A multi - scale analysis of urban form and communiting change in a small metropolitan area (1990 - 2000). The Annals of Regional Science, 41, 2, 315 - 332.

Instituto Nacional de Estadísticas (INE) (2002). Censo Nacional de Población y Vivienda 2002. Recuperado el 10 de enero 2007, de. http://espino.ine.cl/cgibin/RpWebEngine.exe/Port alAction?\&MODE=MAIN\&BASE=CPCHL2KCOM\&MAIN=WebServerMain.inl.

McDonald, J. \& Prather, P. (1994). Suburban employment centres: the case of Chicago. Urban Studies, 31, 2, $201-218$.

Muñiz, I.; Galindo, A. \& García-López, M. (2005). Descentralización, integración y policentrismo en Barcelona. Recuperado el 5 de junio 2007, de http://ideas.repec.org/p/uab/ wprdea/wpdea0505.html.

Muñiz, I.; García-López, M. \& Galindo, A. (2008). The effect of employment sub-centers on population density in Barcelona. Urban Studies, 45, 627-649.

Pauchard, A.; Aguayo, M.; Peña, E. \& Urrutia, R. (2006). Multiple effects of urbanization on the biodiversity of developing countries: the case of a fast - growing metropolitan area (Concepción, Chile). Biological Conservation 127: 272- 281.

Pérez, L. \& Salinas, E. (2007). Crecimiento urbano y globalización: Transformaciones del Área Metropolitana de Concepción, Chile, 1992-2002. Revista Bibliográfica de Geografía y Ciencias Sociales Scripta Nova. Recuperado el 10 octubre 2007, de http://www.ub.es/ geocrit/sn/sn-251.htm.

Redfearn, C. L. (2007). The topography of metropolitan employment: Identifying centers of employment in a polycentric urban area. Journal of Urban Economics, 61, 519-541.

Rojas, J.; Azócar, G.; Vega, C.; Kindler, A. \& Kabish, S. (2006). Atlas social y ambiental del Área Metropolitana de Concepción, Región del Bio-Bio, Chile. Transformaciones sociodemográficas y ambientales 1992 - 2002. Concepción: Universidad de Concepción.

Schwanen, T.; Dieleman, F. M. \& Dijst, M. (2001). Travel behaviour in Dutch monocentric and policentric urban systems. Journal of Transport Geography, 9, 173-186.

Vásquez, A.; Riveros, S. \& Romero, H. (2005). Sustentabilidad del desarrollo urbano del Gran Concepción. XXVI Congreso Nacional y XVI Congreso Internacional de Geografía Sociedad Chilena de Ciencias Geográficas, Pontificia Universidad Católica de Chile. Santiago de Chile.

Wilson, A. (2000). Complex spatial systems: the modelling foundations of urban and regional analysis. Edinburgh: Prentice Hall.

William, A. \& Clark, A. (2000). Monocentric to policentric: new urban form and old paradigms. En G. Bridge \& A. Watson (Eds.), Companion to the city (pp. 141-154). Oxford: Blackwell Publishers. 